MATEC Web of Conferences 42, 01006 (2016)

DOI: $10.1051 /$ matecconf/20164201006

(c) Owned by the authors, published by EDP Sciences, 2016

\title{
Indoor Autonomous Airship Control and Navigation System
}

\author{
Roman Fedorenko ${ }^{\mathrm{a}}$, Victor Krukhmalev \\ Southern Federal University, Russia
}

\begin{abstract}
The paper presents an automatic control system for autonomous airship. The system is designed to organize autonomous flight of the mini-airship performing flight mission defined from ground control station. Structure, hardware and software implementation of indoor autonomous airship and its navigation and control system as well as experiment results are described.
\end{abstract}

\section{Introduction}

Invented over 100 years ago, airships development is highly motivated in present days. Airships can be used as both a passenger and freight transport, as well as tools for patrolling, reconnaissance, monitoring, aerial photography, mapping, advertising and promotion and for communication channels. Unlike fixed wind aircraft and helicopter, which spend about two- thirds of the engine thrust to maintain its weight in air, airships do not need to create the lift force. Hence, they can perform in long ranges with high-duration of non-stop flights. Airships consume energy only on acceleration and overcoming air resistance that means lower propulsion power than for airplane or helicopter with the same payload.

Airships are environmentally friendly. It results from appliance of smaller propulsion plants, consuming lower fuel and therefore having lower exhaust emissions if it is engines and less electromagnetic pollution if it is electric motors. Lower fuel consumption of airships also means a lower cost of the flight. Advantages of airships are obvious. Hence, the development of airships and its performance as universal aerial platforms are highly motivated.

Use of autonomous control systems can eliminate the human factor, increase performance, will allow making long non-stop flights, which is important for problems of patrols [1-3] and for the stratospheric transportation [4-6].

When developing of new control approaches for airship, many flight tests should be performed. Indoor airship is convenient for this needs, because it is cheaper, can be rapidly deployed, etc. However, control of aerial vehicle indoor faces specific difficulties mainly related to organizing of indoor navigation. Rapidly used for this applications motion capture systems are too expensive and requires installation and configuration. Therefore, we tried using visual odometry system and got acceptable results.
Our hardware and software organization of testing autonomous airship for indoor usage and its control system, navigation system, ground control station are described in this paper.

\section{Problem formulation}

Pattern of use of the autonomous airship considered is as follows: ground control station operator visually on the map forms flight mission by the segments (as shown at Fig. 1) and control system calculates the required control (forces and moments and the settings of the actuators) for performing of a given flight task.

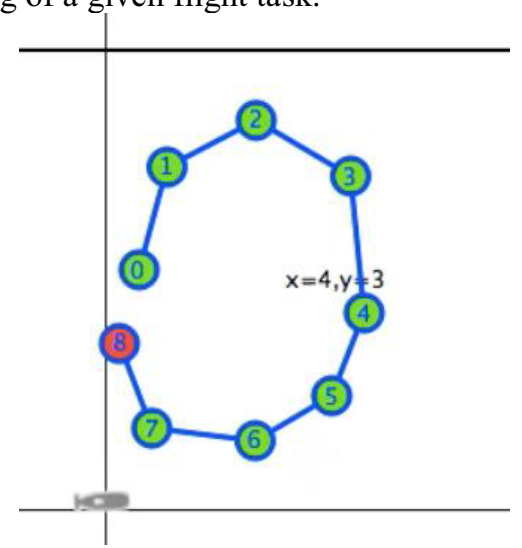

Figure 1. Flight task example

The problem is to construct navigation and control system of airship is to autonomous execution of a given flight task.

\section{Airship specifications}

Airship shown in Figure 2 and its specifications presented in Table 1.

\footnotetext{
a Corresponding author: frontwise@gmail.com
} 


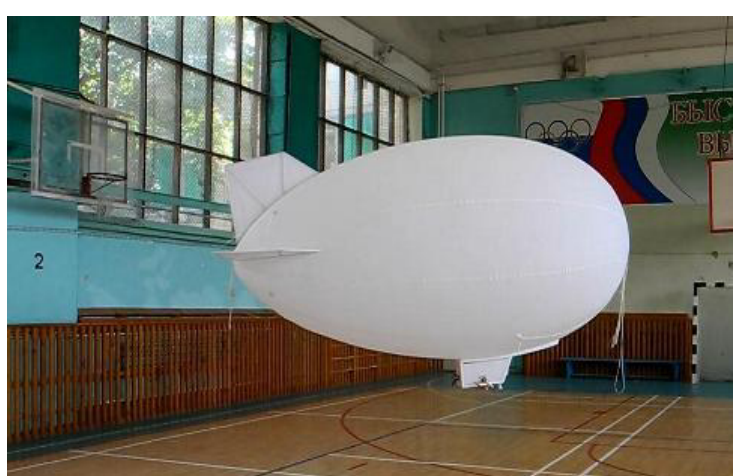

Figure 2. Indoor airship

Table 1 Airship specifications

\begin{tabular}{|c|c|}
\hline Parameter & Value \\
\hline Envelope volume & $5.2 \mathrm{~m} 3$ \\
\hline Length & $3.78 \mathrm{~m} 3$ \\
\hline Envelope mass & $2.5 \mathrm{~kg}$ \\
\hline Equipment mass & $3.7 \mathrm{~kg}$ \\
\hline Buoyant gas & $\mathrm{Helium}$ \\
\hline Inertia moments: & \\
\hline $\mathrm{Jx}$ & $3.67 \mathrm{~kg} \cdot \mathrm{m} 2$ \\
\hline $\mathrm{Jy}$ & $6.27 \mathrm{~kg} \cdot \mathrm{m} 2$ \\
\hline $\mathrm{Jz}$ & $4.63 \mathrm{~kg} \cdot \mathrm{m} 2$ \\
\hline Aerodynamics: & \\
\hline $\mathrm{Cx}$ & 0.011 \\
\hline $\mathrm{Cy}$ & 0.013 \\
\hline $\mathrm{Cz}$ & 0.19 \\
\hline
\end{tabular}

\section{Autonomous airship navigation and control system hardware}

Photos of airship gondola are given at Fig. 3. Autonomous airship navigation and control system hardware structure is shown in Fig. 4. Paradigm of division computing part into high (computer) and low (microcontroller) level used. Microcontroller unit gets data from computer or remote controller and generates PWM signal to motors and servos. Computer calculates required control actions according to control algorithm, runs software part of navigation system, and communicates with ground control station.

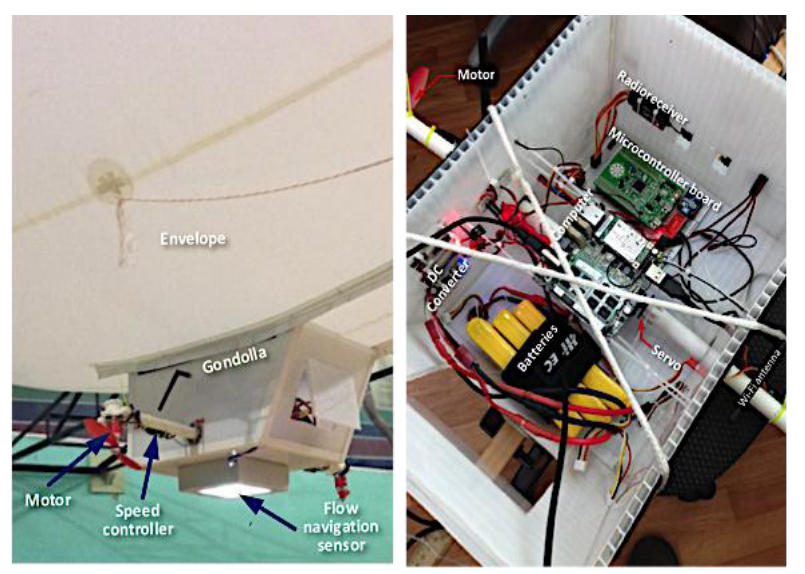

Figure 3. Airship hardware

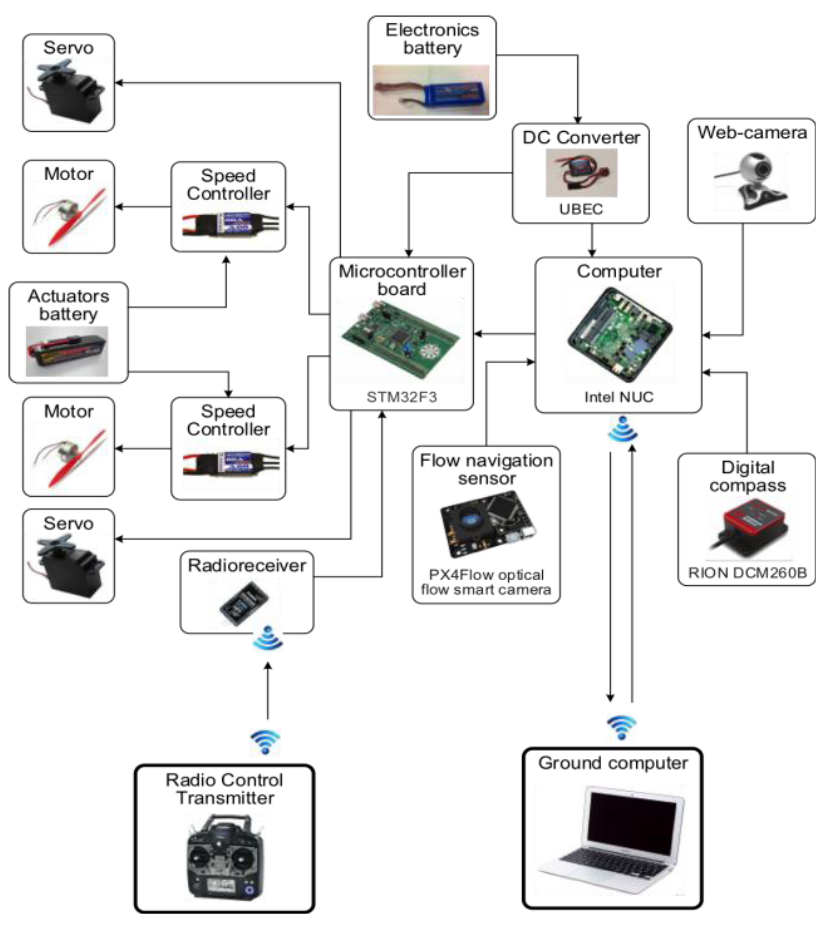

Figure 4. Hardware implementation of autonomous airship structure

\section{Control algorithm and distribution of control forces and moments on the actuators}

Control algorithm is described in [3, 7, 8]. Main advantage of control algorithm is consideration of propulsion drives on the level of control forces and moments. As far as motors are unable to generate $\mathrm{Fz}$ and $\mathrm{Mz}$ forces, control algorithm calculates 4 forces and moments: Fx, Fy, Mx, My.

$$
\begin{aligned}
& A_{3}=\left[\begin{array}{l}
-\psi_{0} \\
-\gamma_{0}
\end{array}\right], A_{4}=\left[\begin{array}{lll}
1 & 0 & 0 \\
0 & 1 & 0
\end{array}\right], A_{5}=\left[\begin{array}{lll}
0 & 0 & 0 \\
0 & k_{y} & 0
\end{array}\right], A_{6}=\left[\begin{array}{c}
-V_{k} \\
-k_{y} y_{z}
\end{array}\right], \Phi \\
& \psi_{t r}=A_{2}\left[\begin{array}{c}
\psi \\
v \\
\gamma
\end{array}\right]+A_{3}, \dot{\psi}_{t r}=A_{2} A_{\omega}\left[\begin{array}{c}
\omega_{x} \\
\omega_{y} \\
\omega_{z}
\end{array}\right], \psi_{V}=A_{4} \bar{V}+A_{5} \bar{X}+A_{6},
\end{aligned}
$$

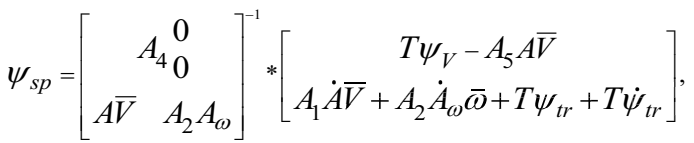

$$
\begin{aligned}
& F_{u}=-M_{c o n}^{-1}\left(M_{d v}\left(F_{d y n}+F_{e x t}\right)+\psi_{s p}\right)
\end{aligned}
$$

Where $\psi_{0}, v_{0}, \gamma_{0}$ are required angles of heading, roll and pitch; $\psi, v . \gamma$ are current angles of heading, roll and pitch; $A_{2}$ is the matrix of ones of required dimension; $A$ is the standard matrix of kinematics rotation, $\dot{A}-$ its time derivative; A $\omega$ is the matrix of dynamics rotation, $\dot{A}_{\omega}$ is its time derivative; $\bar{X}=\left[\begin{array}{lll}x & y & z\end{array}\right]^{T}$ is the vector of linear position of airship; $\bar{V}=\left[\begin{array}{lll}V_{x} & V_{y} & V_{z}\end{array}\right]^{T}$ is the vector of linear velocities, $\bar{\omega}=\left[\begin{array}{lll}\omega_{x} & \omega_{y} & \omega_{z}\end{array}\right]$ is the angular rates vector; $M_{c o n}$ is the common matrix of mass-inertia parameters; $T$ is the time constant, defining convergence of processes; $M_{d v}$ is the matrix of mass-inertia parameters for the given control channels vector $\left[F_{x} F_{y} M_{x} M_{y}\right]^{T} ; F_{d y n}$ is the vector 
of dynamic forces and moments; $F_{\text {ext }}$ is the vector of external forces - buoyancy and gravity; $V_{x}, V_{y}, V_{z}$ are airship velocity vector components; $V_{k}$ is velocity wanted; $y, y^{0}$ are current and target altitudes, $k_{y}$ is proportional coefficient.

For implementation of control forces and moments, one needs to distribute this control vector into propulsion drives. Propulsion drives implement classical zeppelin drives scheme, which shown in Fig. 5. So we have to calculate how to transform general control vector $F_{u}=\left[F_{x}\right.$ $\left.F_{y} M_{x} M_{y}\right]^{T}$ into four actuators state: left motor thrust $P_{l}$, right motor thrust $P_{r}$, left motor tilt angle (left servo position) $\alpha_{l}$, right motor tilt angle (right servo position) $\alpha_{r}$.
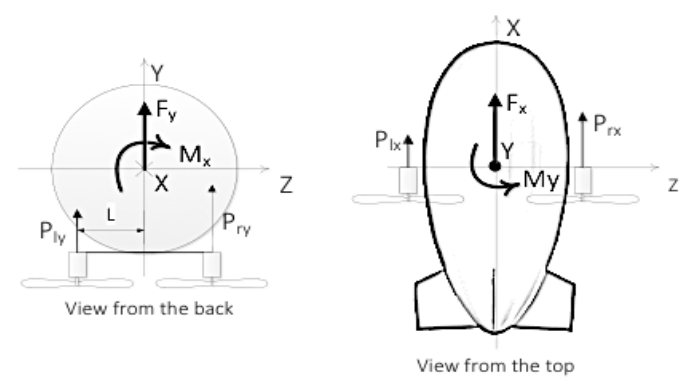

Figure 5. Airship propulsion drives

As we can see in the Fig. 5, motors are of the "pushing" type. Left side of the figure shows drives thrusts as looking to the tail of the airship. The $\mathrm{X}$ axis is directed outward us. $P_{r y}$ is projection of right thrust to the $\mathrm{Y}$-axis and $P_{l y}$ is left thrust projection. $L$ is the lever distance from the point of appliance of force to $\mathrm{Y}$-axis. For simplicity we virtually place point of applying thrust $P_{r y}$ and $P_{l y}$ to the Z-axes (Fig. 5, left side), and we can write for this system:

$F_{y}=P_{l y}+P_{r y}$,

$M_{x}=P_{l y} L-P_{r y} L$

Let us look at the right side of Fig.3. In the horizontal plane, motors' thrust appliance points are located at Zaxis. As far as $\mathrm{Y}$-axis directed toward us, positive $M_{y}$ is anti-clockwise. Hence we can write:

$F_{x}=P_{l x}+P_{r x}$,

$M_{y}=-P_{l x} L+P_{r x} L$

Motors propellers rotate in opposite directions, compensating each other and not generating additional moments. Taking into consideration (2) and (3) the following calculations has to be done in order to get actuators actions $P_{l}, P_{r}, \alpha_{l}, \alpha_{r}$ :

$P_{r x}=0.5 F_{x}+0.5 M_{y} / L$,

$P_{l x}=0.5 F_{x}-0.5 M_{y} / L$,

$P_{r y}=0.5 F_{y}+0.5 M_{x} / L$,

$P_{l y}=0.5 F_{y}-0.5 M_{x} / L$,

$P_{l}=\sqrt{P_{l x}^{2}+P_{l y}^{2}}$,

$P_{r}=\sqrt{P_{r x}^{2}+P_{r y}^{2}}$

$\alpha_{r}=\arctan \left(P_{r y}, P_{r x}\right)$,

$\alpha_{l}=\arctan \left(P_{l y}, P_{l x}\right)$.

Another pure hardware problem is that motors and its hardware controller, which are of-the-shelf products, has its own nonlinear characteristic of PWM signal to generated thrust. So for adequate control we need to know what PWM number means required thrust value. Hence we need to identify this characteristic of PWM signal from thrust. For this purpose motors were connected to thrust measurement device. Thrust and PWM number were measured in 18 points in all motor thrust range $0-8 \mathrm{~N}$. Resulting polynomial for one motor, while both of motors are equal, is:

$S_{P W M}=p_{1} F^{2}+p_{2} F+p_{3}$,

where $\mathrm{p}_{1}, \mathrm{p}_{2}, \mathrm{p}_{3}$ are polynomial coefficients, calculated by classical regression analysis, presented in Table $2 ; \mathrm{F}$ is a value of current required thrust.

Table 2. Polynomial coefficients

\begin{tabular}{|c|c|}
\hline $\begin{array}{c}\text { Polynomial } \\
\text { coefficient }\end{array}$ & Value \\
\hline $\mathrm{p} 1$ & -0.282124607447971 \\
\hline $\mathrm{p} 2$ & 11.0206645912410 \\
\hline $\mathrm{p} 3$ & 115.129041258376 \\
\hline
\end{tabular}

Polynomial plot shown in the Figure 4 with solid blue line. Red round markers show measured values of thrust and PWM number.

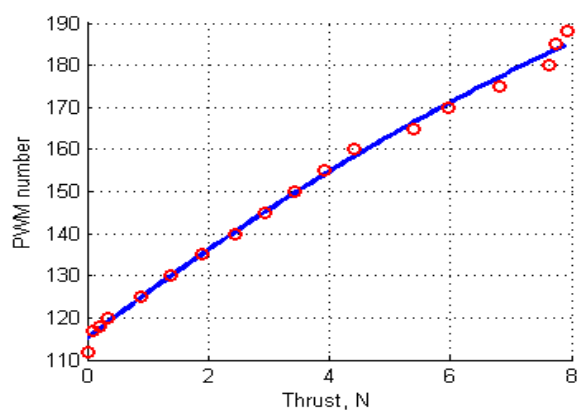

Figure 6. Dependence of motor-controller PWM from motor thrust

\section{Indoor navigation system}

It is supposed that current values of coordinates, linear velocities, angular rates are known for Control algorithm (1). These data are called navigation data, and should be provided by navigation system. Navigation indoor is not trivial task due to the invalidity of GPS system. For this reason, navigation is based on Rion DCM260B electronic 3D compass (Figure 7) and PX4FLOW optical flow smart camera (Figure 8).

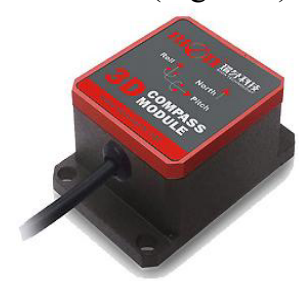

Figure 7. Rion DCM260B electronic 3D compass

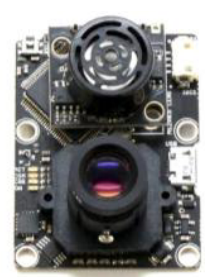

Figure 8. PX4FLOW optical flow smart camera 
Optical flow smart camera data contains timestamp (with sequence number and frame id), ground distance (measured by ultrasonic ranging module), lateral and longitudinal velocities of blimp in $\mathrm{m} / \mathrm{s}$ (and in pixels in flow_ fields) and quality field (which shows measurement correctness depended mainly on lighting conditions).

Airship indoor navigation uses this data to produce airship pose and velocity data in a way, shown at Fig. 9.

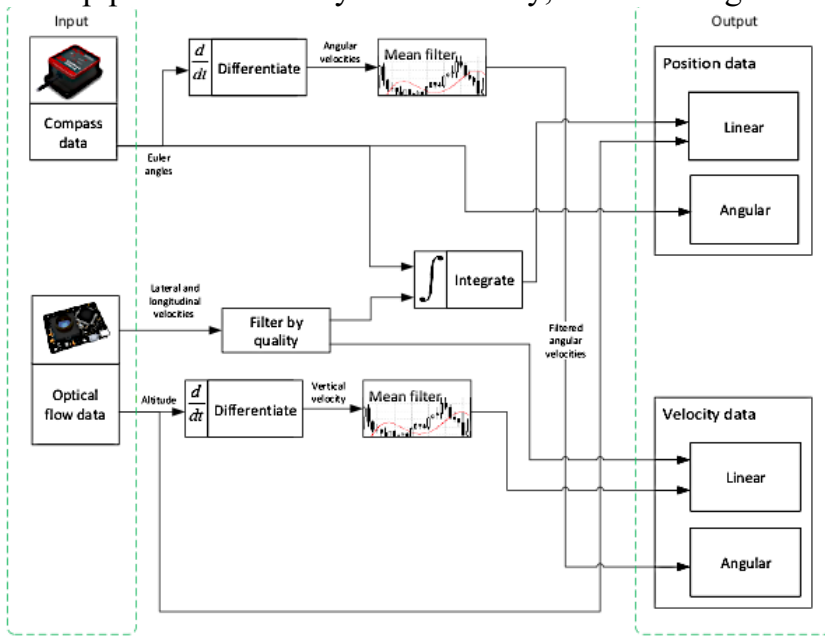

Figure 9. Blimp navigation data flow diagram

To transform lateral and longitudinal velocities of blimp in body coordinate frame to earth-fixed coordinate frame following equation is used:

$$
\begin{aligned}
& d x=-V_{x}{ }^{*} \cos (\psi)-V_{y}{ }^{*} \sin (\psi) ; \\
& d z=-V_{x}{ }^{*} \sin (\psi)+V_{z}{ }^{*} \cos (\psi) ;
\end{aligned}
$$

where $\psi$ is yaw field from compass data, converted to radians.

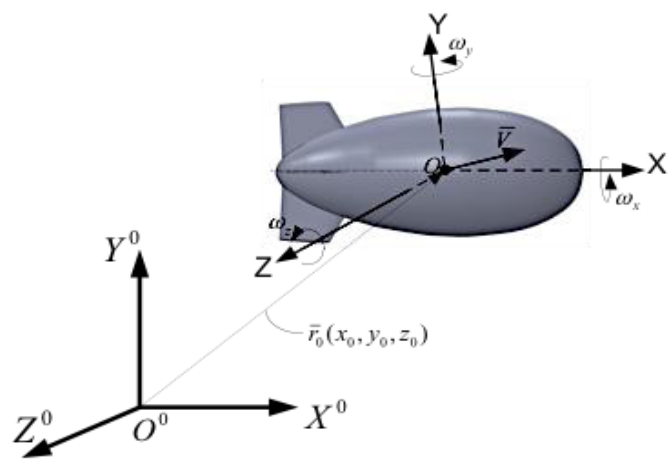

Figure 10. Axes orientation

After this coordinate frames conversion integration performed to get airship coordinates in ground frame:

$$
\begin{aligned}
& x+=d x * d t ; \\
& z+=d z * d t ;
\end{aligned}
$$

This is what Integrate block at Fig. 9 does.

To get angular velocities having only angles at every moment and vertical speed having altitude we differentiate this data and use moving average filter to smooth data.

\section{Control system software implementation}

Linux Ubuntu operating system used in onboard computer. All the software build in frame of Robot Operating System [9], demonstrating sparse module interconnection, client-server and publisher-topicsubscriber paradigms. Structure is shown in Fig. 11. Oval structures show processes. Small rectangular structures are topics.

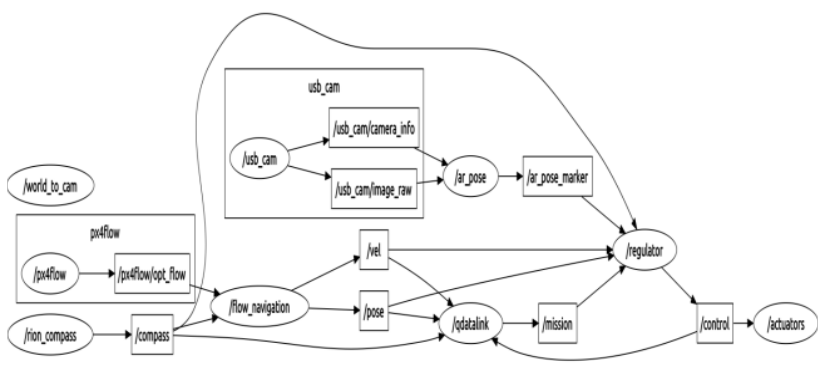

Figure 11. Software structure

Main software nodes (processes) are:

/regulator - performing actuators control calculation based on navigation data (/vel, /pose and /compass topics) and mission (/mission topic);

/flow_navigation - performing visual odometry navigation based on PX4FLOW camera data (//px4flow/opt flow topic) and orientation data (/compass topic);

/actuators - realizing data communication protocol with microcontroller board to send generated controls from regulator (from /control topic);

/qdatalink - realizing communication with ground control station based on MAVLink protocol to get flight task (publishing it at /mission topic) and send telemetry data (from (/vel, /pose /compass and /control topics) to ground control station;

/ar_pose - QR-code-based visual navigation (for further experiments).

\section{Experiments}

Visual odometry based indoor navigation system was tested first. Fig. 12 shows measured coordinates of flow sensor (black) moved on mobile platform with fixed heading and near-zero pitch and roll angles along $5 \times 5 \mathrm{~m}$ square and $4 \times 1 \mathrm{~m}$ oval trajectories (red). Results shown demonstrate ability to make autonomous indoor airship flight with this navigation system.
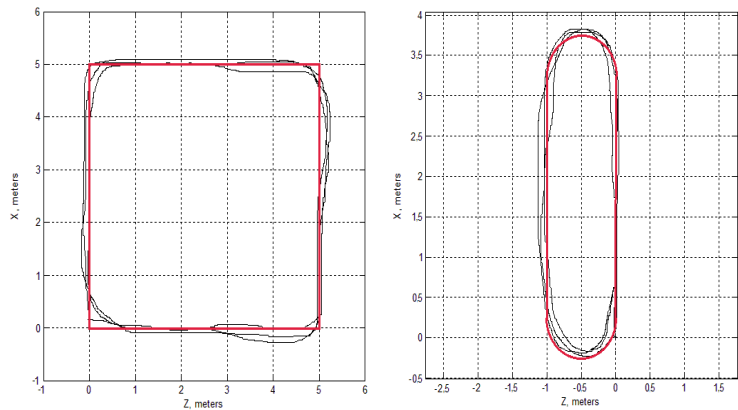

Figure 12. $5 \times 5 \mathrm{~m}$ square and $4 \times 1 \mathrm{~m}$ oval trajectories of flow sensor (red) and measured coordinates (black) 
Results of autonomous flight shown in Fig. 13 - Fig. 18. From the ground computer 8 waypoints were given, as it is shown in down right corner of Fig. 13. Flight mission is to go through these points in serial order.

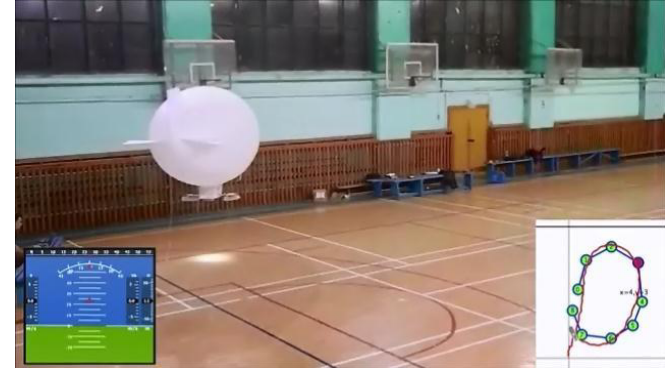

Figure 13. Airship autonomous flight

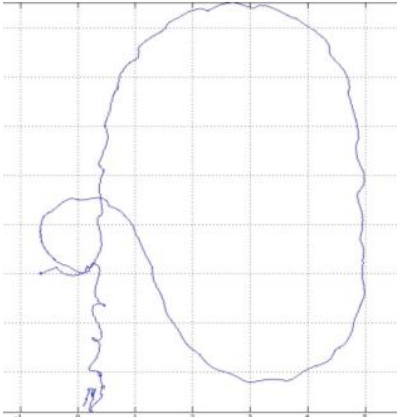

Figure 14. Trajectory of flight, dimensions are meters

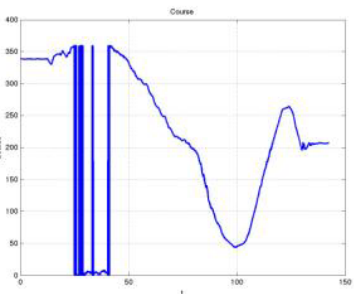

Figure 16. Course angle (deg) against time (s)
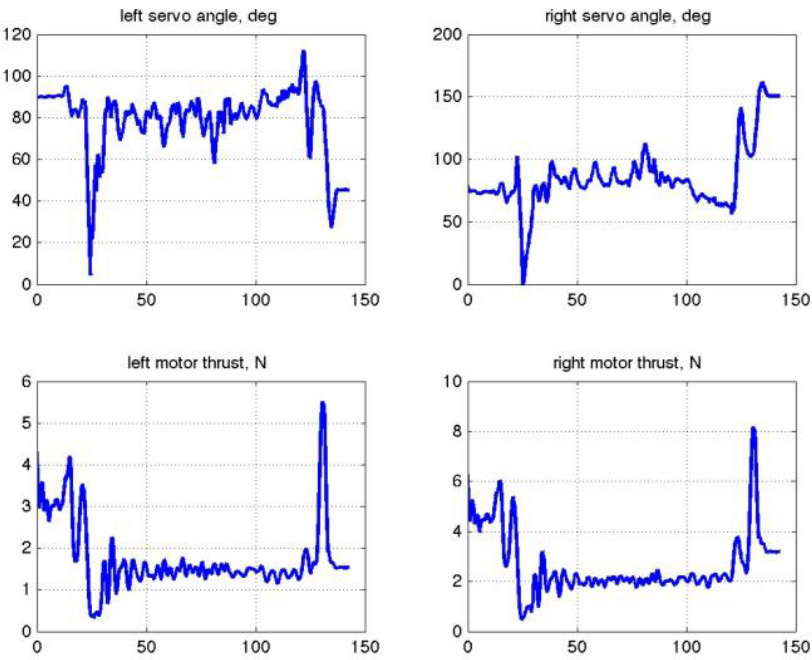

Figure 18. Actuators controls against time (s)

Additional experiments results with another flight tasks shown at Fig. 19.

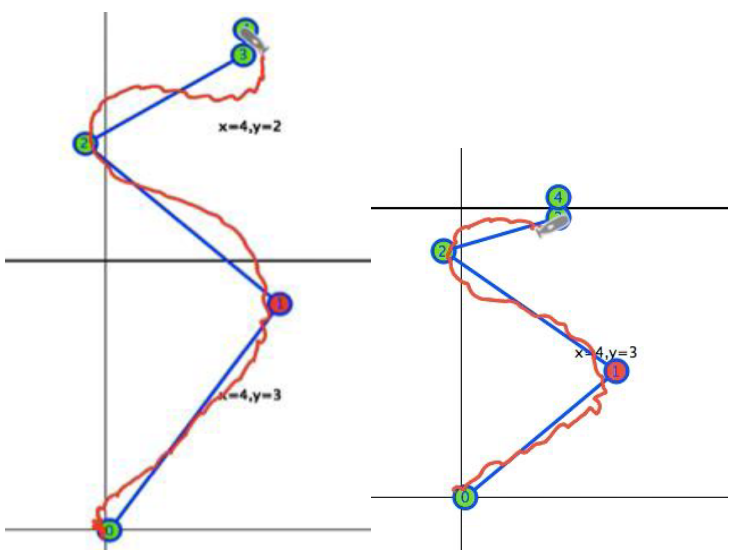

Figure 19. Additional experiments results

\section{Conclusion}

However, control of aerial vehicle indoor faces specific difficulties mainly related to organizing of indoor navigation. Cheap indoor navigation system development approach shown in this paper yielded acceptable results. So further experiments concerned the control system based on position-trajectory control law. Control system shows good results despite of some oscillation, which took place due too big overweighting of airship.

Control system structure, hardware and software developed allows performing further tests with different control and planning algorithms indoor.

\section{Acknowledgments}

Authors are deeply grateful to their scientific supervisor Prof. Viacheslav Pshikhopov.

The presented work is performed as part of the Multibody Advanced Airship for Transport (MAAT) project with ref. 285602, supported by European Commission through the 7th Framework Programme, and which is gratefully acknowledged.

This work was supported by the Russian Ministry of Education and Science, research No114041540005 "Theory and methods of position-trajectory control of maritime robotic systems in extreme conditions and under uncertainty environment", grant from the Southern Federal University "Theory and methods for energyefficient control of distributed systems of generation, transmission and consumption of electricity" and Grant of the president of Russian Federation for state support of the Russian leading scientific school SSc-3437.2014.10.

\section{References}

1. Pshikhopov, V., Medvedev, M., Neydorf, R., Krukhmalev, V. et al., "Impact of the Feeder Aerodynamics Characteristics on the Power of Control Actions in Steady and Transient Regimes," SAE Technical Paper 2012-01-2112, 2012, doi:10.4271/2012-01-2112.

2. Pshikhopov, V., Krukhmalev, V., Medvedev, M., and Neydorf, R., "Estimation of Energy Potential for 
Control of Feeder of Novel Cruiser/Feeder MAAT System," SAE Technical Paper 2012-01-2099, 2012, doi:10.4271/2012-01-2099.

3. Pshikhopov, V., Medvedev, M., Kostjukov, V., Fedorenko, R., Gurenko, B., Krukhmalev, V. "Airship Autopilot Design," SAE Technical Paper 2011-01-2736, 2011, doi:10.4271/2011-012736

4. Pshikhopov, V.Kh., Krukhmalev, V.A., Medvedev, M.Yu., Budko, A.Yu., Chufistov, V.M., Adaptive control system design for robotic aircrafts, 2013 IEEE Latin American Robotics Symposium, LARS 2013, doi:10.1109/LARS.2013.59

5. Pshikhopov, V.Kh., Medvedev, M.Yu., Block design of robust control systems by direct Lyapunov method, 2011, IFAC Proceedings Volumes (IFAC-
PapersOnline), doi: 10.3182/20110828-6-IT1002.00006

6. Pshikhopov, V.Kh., Ali, A.S., Hybrid motion control of a mobile robot in dynamic environments, 2011, IEEE International Conference on Mechatronics, ICM 2011 - Proceedings

7. Pshikhopov, V.Kh., Medvedev, M.Yu, Robust control of nonlinear dynamic systems, 2010, IEEE ANDESCON Conference Proceedings, ANDESCON 2010

8. Neydorf, R., Krukhmalev, V., Kudinov, N., and Pshikhopov, V., "Methods of Statistical Processing of Meteorological Data for the Tasks of Trajectory Planning of MAAT Feeders," SAE Technical Paper 2013-01-2266, 2013, doi:10.4271/2013-01-2266

9. http://www.ros.org 